\title{
NRO Legacy Project: M33 all Disk Survey of Giant Molecular Clouds with NRO 45-m and ASTE 10-m telescopes
}

\author{
N. Kuno ${ }^{1,2}$, T. Tosaki ${ }^{3}$, S. Onodera ${ }^{1}$, R. Miura ${ }^{4}$, K. Muraoka ${ }^{5}$, \\ S. Komugi ${ }^{6}$, T. Sawada ${ }^{6}$, K. Nakanishi ${ }^{6}$, K. Kohno ${ }^{7,8}$, H. Kaneko ${ }^{1,2}$, \\ A. Hirota ${ }^{1}$, N. Arimoto ${ }^{2,6}$, H. Nakanishi ${ }^{9}$, and R. Kawabe ${ }^{1}$ \\ ${ }^{1}$ Nobeyama Radio Observatory, National Astronomical Observatory of Japan, \\ Minamimaki, Minamisaku, Nagano, 384-1305, Japan \\ ${ }^{2}$ The Graduate University for Advanced Studies (SOKENDAI), \\ Osawa, Mitaka, Tokyo, 181-8588, Japan \\ ${ }^{3}$ Joetsu University of Education, Yamayashiki-machi, Joetsu, Niigata, 943-8512, Japan \\ ${ }^{4}$ University of Tokyo, Department of Astronomy, School of Science, \\ Hongo, Bunkyo, Tokyo, 113-0033, Japan \\ ${ }^{5}$ Department of Physical Science, Osaka Prefecture University, \\ Gakuen 1-1, Sakai, Osaka, 599-8531, Japan \\ ${ }^{6}$ National Astronomical Observatory of Japan, Osawa, Mitaka, Tokyo, 181-8588, Japan \\ ${ }^{7}$ Insititute of Astronomy, School of Science, The University of Tokyo, \\ Osawa, Mitaka, Tokyo, 181-0015, Japan \\ ${ }^{8}$ Research Center for Early Universe, School of Science, The University of Tokyo, \\ Hongo, Bunkyo, Tokyo, 113-0033, Japan \\ ${ }^{9}$ Graduate School of Science and Engineering, Kagoshima University, \\ 1-21-35 Korimoto, Kagoshima, Kagoshima 890-0065, Japan
}

\begin{abstract}
We have conducted all disk imaging of $\mathrm{M} 33$ in ${ }^{12} \mathrm{CO}(1-0)$ using the $45-\mathrm{m}$ telescope at Nobeyama Radio Observatory. We present preliminary results of this project. The spatial resolution of $\sim 80 \mathrm{pc}$ is comparable to the size of GMCs. The identified GMCs show wide variety in star forming activity. The variety can be regarded as the difference of their evolutionary stage. We found that Kennicutt-Schmidt law breaks in GMC scale $(\sim 80 \mathrm{pc})$, although it is still valid in $1 \mathrm{kpc}$ scale. The correlation between molecular gas fraction, $f_{m o l}=\Sigma\left(\mathrm{H}_{2}\right) / \Sigma\left(\mathrm{HI}+\mathrm{H}_{2}\right)$ and gas surface density shows two distinct sequences and shows that $f_{m o l}$ tends to be higher near the center. We also made partial mapping ${ }^{12} \mathrm{CO}(3-2)$ with ASTE telescope. These data show that the variation of physical properties of molecular gas are correlated with the GMC evolution and mass. That is, GMCs with more active star formation and more mass tend to have higher fraction of dense gas.
\end{abstract}

Keywords. galaxies: individual(M33), ISM, GMC

\section{Introduction}

Since M33 is one of the nearest spiral galaxies in the local group and moderately face-on, the galaxy is suitable to resolve each GMCs and investigate their properties. Furthermore, there are many other frequency data of tracers of atomic gas, star forming regions, and stars, for comparisons. Therefore, M33 is the best target for studying GMCs and star formation within a whole galaxy. Our observations are performed with Nobeyama 45m and ASTE telescopes. The 25 multi-beam receiver, BEARS, mounted 
on the $45 \mathrm{~m}$ telescope and CATS345 installed in ASTE were used. We can get ${ }^{12} \mathrm{CO}(1-0)$ and ${ }^{12} \mathrm{CO}(3-2)$ data with highly uniform quality by using these receivers and On-The-Fly technique. Our scientific objectives are to investigate the basic properties of GMCs and their spatial variation, and to understand the evolutionary process of GMCs and massive star formation. We will make GMC catalog with the information about their evolutionary stage using our data and other frequency data (e.g., optical data with SUBARU). For these purposes, we have to observe the variation of the internal structure of GMCs along their evolutionary stage eventually. Observations of the internal structure of GMCs in M33 will be achieved by ALMA.

\section{Preliminary results}

Fig. 1 shows the total integrated intensity map of ${ }^{12} \mathrm{CO}(1-0)$ and intensity weighted mean velocity map. Many GMCs are identified in the map and global galactic rotation can be seen. Fig. 2 shows the comparison between $\mathrm{CO}$ and $\mathrm{HI}$, and $\mathrm{CO}$ and star formation rate. We can see the variation of evolutionary stage of GMCs in fig. 2. Namely, there are some GMCs with no star forming activity and HII regions without associated GMCs. GMCs are located on the HI peaks in the outer region, but not in the inner region. We present some preliminary results of our project below.

\subsection{Breakdown of Kennicutt-Schmidt law}

Kennicutt-Schmidt law is a well-known correlation between surface gas density and star formation rate in galaxies. But, it is not clear in what scale the Kennicutt-Schmidt law breaks down. To answer the question, Onodera et al. (2010) compared the surface density of molecular gas and that of the star formation rate in various spatial resolution (fig. 3). These plots show that the correlation is evident in $1 \mathrm{kpc}$ and $500 \mathrm{pc}$ resolution. The correlation becomes looser with higher resolution and finally breaks down in 80 pc resolution. These results indicate that the resolution of $80 \mathrm{pc}$, which is comparable to GMC scales, is the scale in which the Kennicutt-Schmidt law becomes invalid. The comparison between distributions of $\mathrm{CO}$ and star forming regions implies that the main cause of the breakdown is that the variation of evolutionary stage of GMCs becomes apparent in this scale as seen in fig. 2 .

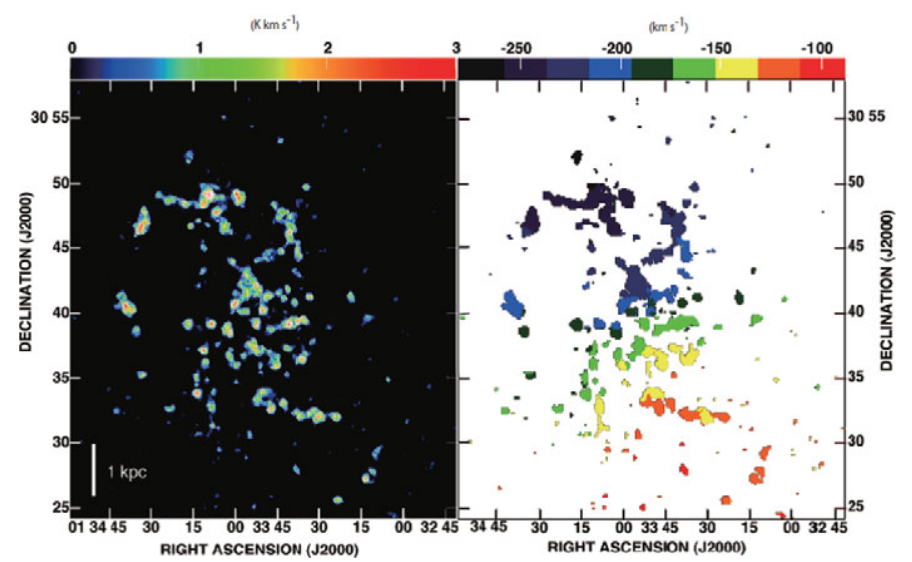

Figure 1. ${ }^{12} \mathrm{CO}(1-0)$ integrated intensity map of M33 (left) and velocity field (right). (Tosaki et al. 2011) 


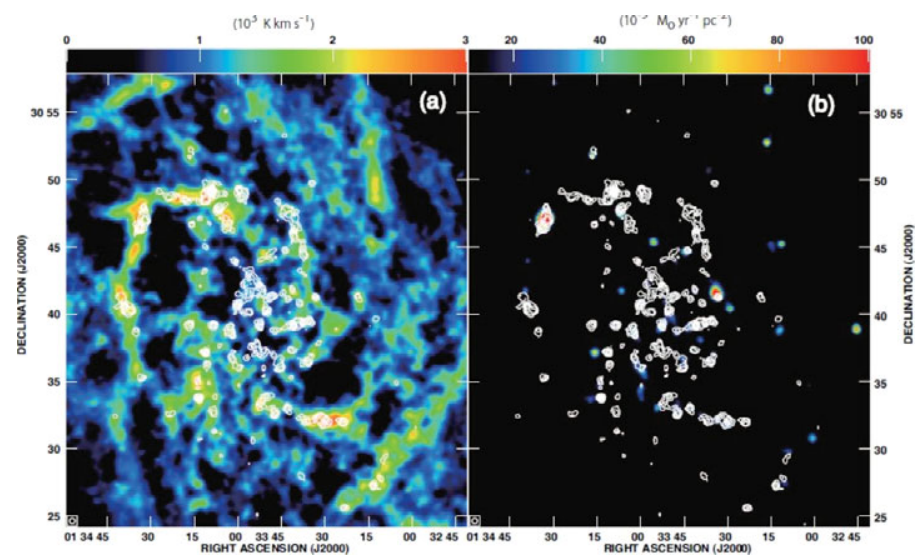

Figure 2. The ${ }^{12} \mathrm{CO}(1-0)$ map superposed on HI (Rosolowsky et al. 2007) (left) and star formation rate derived from $\mathrm{H} \alpha$ luminosity (Hoopes and Walterbos 2000) with extinction-correction using $24 \mu \mathrm{m}$ data (Rieke et al. 2004) (right). (Tosaki et al. 2011)

\subsection{HI to H2 transition}

Tosaki et al. (2011) make comparison between the CO and HI maps and show that molecular gas fraction $f_{m o l}$ is higher in the inner region than in the outer region (fig. 2). Fig. 4 shows correlation between gas surface density and $f_{m o l}$. It is apparent that there
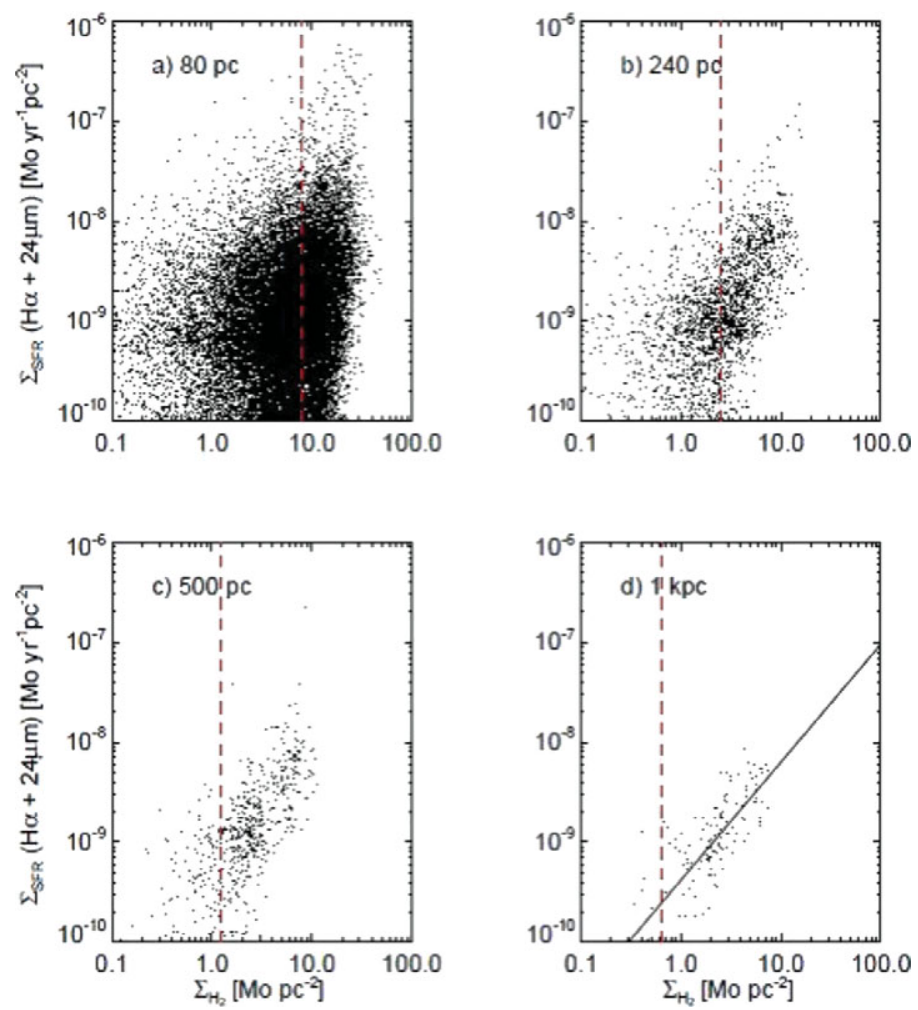

Figure 3. Star formation rate per unit area vs. surface density of $\mathrm{H}_{2}$ gas for four different resolutions. The broken lines represent $2 \sigma$ of surface density of $\mathrm{H}_{2}$. The line in d) shows the best fit to the $\sim 1 \mathrm{kpc}$ resolution data. (Onodera et al. 2010) 


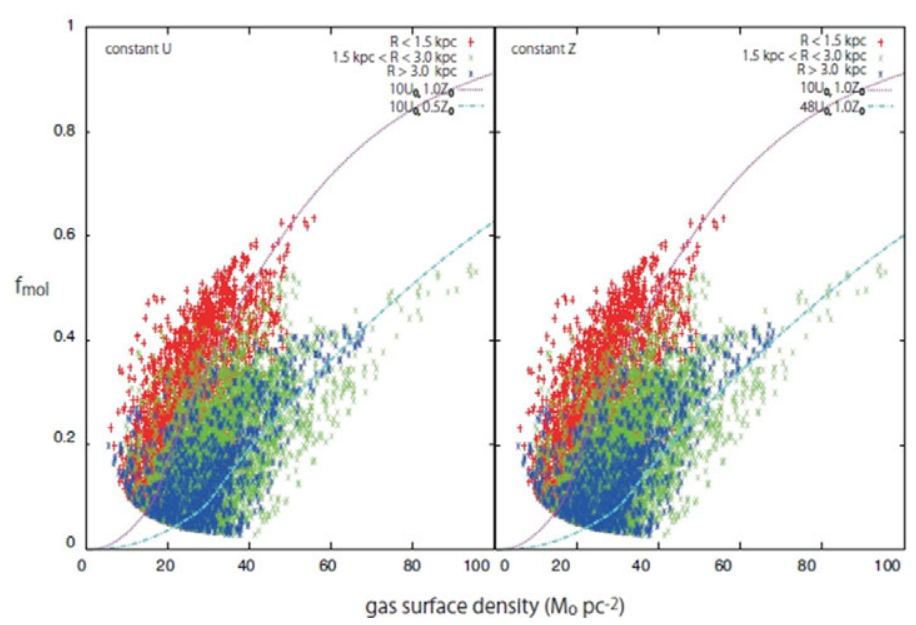

Figure 4. Correlation between gas surface density and $f_{m o l}$. Data of $R \leqq 1.5 \mathrm{kpc}, 1.5 \mathrm{kpc}$ $\leqq R \leqq 3 \mathrm{kpc}$, and $R \geqq 3 \mathrm{kpc}$. Dotted and dashed curves indicate correlation for $U=10 U_{0}$ in the case of $Z=Z_{0}$ and $Z=0.5 Z_{0}$ (left), and for $Z=1.0 Z$ in the case of $U=10 U_{0}$ and $U=48 Z_{0}$ (right), respectively. (Tosaki et al. 2011)

are two distinct sequences depending on the distance from the galactic center. $f_{m o l}$ is higher in the inner region $R<1.5 \mathrm{kpc}$ than in the outer region at the same gas surface density. Most probable cause of the difference of $f_{m o l}$ is high metallicity in the inner region. Actually, sharp increase of metallicity in the central $1 \mathrm{kpc}$ region in M33 has been reported by Vilchez et al. (1988). On the other hand, if we attribute the difference to the variation of radiation field, radiation field has to increase with distance from the center. But, radial distribution of tracers of stars and star forming regions does not show such a trend. Another possibility is variation of gas volume density. In fig. 4, gas surface density is used as an indicator of gas volume density assuming that the scale height is constant. But, if the scale height is larger in the outer region than in the inner region as seen in our Galaxy, volume density is higher in the inner region at the same surface density.

\subsection{Variation of $C O(1-0) / C O(3-2)$ ratio in $G M C s$}

Onodera (2009) and Onodera et al. (2011) investigated the variation of $\mathrm{CO}(1-0) / \mathrm{CO}(3-2)$ ratio in GMCs and its dependence on the GMC properties. They show that the ratio depends on star forming activity in GMCs and GMC mass. Namely, GMCs with more active star formation and more massive tend to have higher fraction of dense gas.

\section{References}

Hoopes, C. G. \& Walterbos, R. A. M. 2000, ApJ, 541, 597

Onodera, S. 2009, PhD thesis, University of Tokyo

Onodera, S., et al. 2010, ApJ(Letters) 722, L127

Onodera, S., et al. 2011, in prep.

Rieke, G. H., et al. 2004, ApJS, 154, 25

Tosaki, T., et al. 2011, PASJ, submitted

Vilchez, J. M., et al. 1988, MNRAS, 235, 633 